\title{
MYTHE ET RELIGION DANS \\ LA CANTATA PROFANA DE BÉLA BARTÓK \\ OU LA MYSTÈRE DES FILS \\ CHANGÉS EN CERFS ${ }^{1}$
}

\author{
AMBRUS MISKOLCZY \\ Professeur à l’Université ELTE \\ amiskolczy@gmail.com
}

\begin{abstract}
Les sept fils d'un homme sont changés en cerfs. Ils refusent de quitter la forêt où se trouve la source pure à laquelle ils s'abreuvent. La Cantata Profana, inspirée $\mathrm{du}$ folklore roumain, mais largement retravaillée par Bartók, suggère plusieurs interprétations possibles, fondées sur l'analyse des mythes et et sur l'interprétation des contes, notamment à la manière proposée par Georg Lukács, dont Bartók fut, d'ailleurs, un admirateur circonspect. Les relations tourmentées de Bartók avec le christianisme, particulièrement avec le catholicisme, placent en outre son oeuvre dans une perspective supplémentaire, dont l'horizon est la nature.
\end{abstract}

Mots-clefs : Béla Bartók, Cantata Profana, mythologie, Georg Lukács, analyse psychanalytique du conte, relations magyaro-roumaines

Cantata profana - profession de foi de Béla Bartók. Cette œuvre, y compris dans son titre, un oxymore, se prête particulièrement à l'analyse des interférences entre la modernité et la religiosité. Si l'intrigue de cette Cantata est simple, son histoire, en revanche, y compris celle de sa naissance, et son message sont complexes.

Voici l'intrigue : un vieux chasseur n'avait appris à ses neuf fils nul autre métier que celui de chasseur. Un jour qu'ils poursuivaient un cerf miraculeux, les neuf fils, traversant un pont, furent eux-mêmes changés en autant de cerfs. S'étant mis à leur recherche, le père les retrouva, buvant à une source. Aussitôt, il les visa de son arc, mais l'aîné de ses fils se fit reconnaître par son père et menaça celui-ci de le mettre en pièces. Le père les pria de revenir à la maison où leur mère les attendait. Mais les jeunes cerfs refusèrent l'invitation, affirmant qu'ils n'étaient pas en mesure d'y répondre, car leurs bois ne pourraient pas franchir la porte et aussi parce qu'ils ne buvaient désormais que de la source d'eau pure. Cette mention de la source pure est devenue, d'une part, un impératif catégorique dans l'imaginaire hongrois, d'autre part la boîte de Pandore d'un discours sans fin.

C'est pourquoi le memento lancé par Arthur Schnitzler me semble d'actualité, quand il affirmait que face à une œuvre, il est préférable de se demander ce que 
celle-ci peut nous dire, au lieu de chercher ce que l'on pourrait lui dire à elle. Observons avec regret, toutefois, que cette opposition exclut le dialogue avec l'œuvre, de même que toute discussion plus ou moins pacifique à son sujet.

À propos de la Cantata profana, l'opinion la plus marquante a été formulée par Ferenc Tallián. Le titre de son livre, devenu classique - Cantata profana. Le mythe de la transition -, pose lui-même les bases de ses conceptions sur l'oeuvre. Il s'agirait de la transition organique d'un mythe initiatique et ancestral vers un mythe artistique et individuel. «Bartók, dans le texte de la Cantata, a identifié le noyau mythologique commun à toutes les religions dogmatisées et à toutes les théories sociales visant à investir l'homme d'une croyance en une vie d'ordre supérieure "; « ce noyau est dans le même temps une "religion" et la négation de la religion"; en ultime analyse, " la rigueur de sa forme [celle de la Cantata] suggère l'essence commune de toute religion : credo quia absurdum ${ }^{2}$. »

Mais la question suivante se pose : avons-nous affaire à un rite initiatique à deux étages, en vue d'atteindre une forme nouvelle de transcendance ? La réponse ne peut être qu'affirmative : tandis que les interprétations de détails se confrontent sous le signe d'un provisoire éternel, l'œuvre, quant à elle, demeure dans la sphère de l'inaccessibilité. Aussi, quand le fils du compositeur, Péter Bartók, s'est mis en quête d'un portrait de son père en une seule phrase, il est tombé sur le texte de la Cantata: les fils changés en cerfs ont trouvé le chemin de la vérité en quittant à jamais le monde faux et artificiel de l'homme soi-disant civilisé, et ils ne boivent désormais d'eau « qu'à la source pure ${ }^{3}$ ».

Le texte originel de la Cantata est roumain. En 1914, Bartók a découvert deux chant de Noël (colinda), l'un à Urisiu-de-Sus, l'autre à Idicel, au pied des Carpathes orientales. Seize ans plus tard, il en a fait une synthèse et a prié un poète alors très à la mode, József Erdélyi, dont le père était roumain, de la traduire en hongrois. C'est cette traduction qui a été publiée, en 1930, en tant que « ballade populaire de la collection de Béla Bartók ». Du reste, n'étant pas satisfait de ce travail, le compositeur a par la suite réalisé lui-même une nouvelle traduction qui s'avère être un poème original en raison de trois innovations.

La première innovation est fondée sur un malentendu. Il s'agit du pont sur lequel seraient passés les fils avant d'être changés en cerfs. Il apparaît que Bartók a commis une erreur, car il a noté l'expression roumaine populaire «p-unde » (en fr. : par où) comme punte (en fr. : pont). Ultérieurement, il a corrigé l'erreur, mais seulement dans l'édition de la colinda publiée en allemand et en anglais. Dans la collection achetée par l'Académie Roumaine, on retrouve la forme erronée. Et c'est ainsi qu'elle a échappé même à la vigilance de Constantin Brăiloiu. Plus tard, dans les années 1960, elle a été remarquée par un de ses disciples, Ioan Nicola, qui a écrit un article important sur les colinda portant sur le thème de la métamorphose des fils en cerfs. Or, reprochant à son auteur l'exagération des mérites de Bartók ainis que la valorisation excessive de l'idée de liberté, la revue Steaua 
de Cluj (aujourd'hui Cluj-Napoca) en refusa la publication. Dès lors, la découverte de Nicola est restée ensevelie dans les pages d'une revue de musicologie ${ }^{4}$.

La deuxième innovation de Bartók tient au fait qu'il a traduit le " grand cerf » de la colinda en « cerf miraculeux ». À vrai dire, l'expression de cerf miraculeux ne dit pas grand chose aux Roumains. Au contraire, dans l'imaginaire hongrois, cet animal psychopompe tient une place privilégiée : par cette formule, Bartók a nationalisé le poème.

La troisième innovation est la source pure. Dans le texte roumain, il est dit simplement que les cerfs boivent à la source. Dans la Cantata de Bartók, ils ne boivent que « de la source pure ». Et ainsi triomphe l'idée de pureté. C'est le secret du succès de cette formule. Au demeurant, dans les premières esquisses de Bartók, la source n'est pas " pure », mais « froide », puis l'inspiration a vaincu. Peut-être est-ce mieux ainsi. La Cantata aurait, qui sait ? peut-être inspiré aujourd'hui une réclame pour de l'eau minérale froide.

Ces trois innovations de Bartók sont à l'origine d'une série de spéculations mythologisantes plus ou moins brûlantes. Le compositeur, quant à lui, est toujours resté froid. Il n'a jamais commenté son œuvre. Mais il l'a récitée, il l'a aussi nommée « ma profession de foi la plus profonde » et, en 1936, il a écouté avec émotion sa première représentation à Londres. C'est seulement deux ans plus tard qu'eut lieu la première représentation à Budapest. Un décalage peut-être tactique. L'atmosphère plus ou moins antiroumaine dans le pays dictait sans doute cette précaution, peut-être exagérée. Car même la première traduction du texte roumain par Bartók n'était pas restée sans un écho favorable. Mihály Babits, autorité incontestée de la vie littéraire, y avait trouvé l'apologie de la liberté : pour lui, les cerfs symbolisaient les poètes persécutés par leur patrie ${ }^{5}$. Naturellement, il s'agit d'une projection et d'une soif d'authenticité altérée par le folklore, qui, plus tard, allait même être instrumentalisée par les mouvements d'extrême-droite, source de malentendus ultérieurs, de la part d'un Adorno lui-même qui a fini par suspecter l'œuvre de Bartók.

Comment expliquer l'obsession de Bartók pour le folklore? Que cherchait-il dans le monde des paysans? La réponse la plus simple est correcte : des formes primitives. Confrontés aux antagonismes nationalistes, il a déposé le témoignage suivant, à propos de l'origine d'une mélodie qu'il a appelée "chant long" : " Jusqu'ici, j'ai constaté que cette mélodie s'est propagée en Perse, en Irak, au coeur de l'Algérie, dans l'ancien royaume roumain et en Ukraine, donc chez quatre nationalités différentes. Il est peu probable que ces quatre peuples aient inventé la même mélodie indépendamment les uns des autres. Mais si l'on suppose l'existence d'un processus d'évolution, il faut dès lors accorder la priorité à l'un de ses peuples. Quel chercheur oserait agir ainsi ? [...] Dans ce cas, les pays offensés interdiraient à ce chercheur de mettre le pied sur leur territoire. Je suppose que si nous avions à notre disposition assez de matériel folklorique, les mu- 
siques populaires du monde entier pourraient être réduites à quelques formes originaires, aux types originaires, aux styles originaires. Mais il est évident, qu'on ne pourrait arriver à cet objectif qu'à la condition de produire moins d'armes et de dépenser plus pour les études musicales avant la disparition totale du folklore6. »

Le vrai modèle, pour Bartók, était la nature : « Nous composons en imitant la nature, car la musique paysanne est un phénomène naturel ${ }^{7}$. » La nature, bien sûr, comme source d'inspiration et non comme un terrain à exploiter. En ce qui concerne la Cantata profana - a écrit Bartók dans une lettre adressée en 1931 à son ami roumain, Octavian Beu - en elle, " seulement le texte est roumain, le matériel thématique est de ma composition personnelle, elle n'est pas une imitation de la musique populaire roumaine, la voix populaire est absente même dans les détails. Cette œuvre peut être considérée comme la musique du texte d'une colinda roumaine ${ }^{8}$. » Voilà une vérité partielle. Car Bartók, par ses innovations textuelles, a aussi radicalement altéré le message d'origine.

Il faut donc admettre qu'il y a deux messages distincts : l'un est celui de la colinda (ou des colinda), l'autre est suggéré par le poème de Bartók.

Selon plusieurs auteurs faisant autorités en matière de folklore, le sens originaire des colinda roumaines demeure inconnu. Tout de même, les exégèses évoquent l'idée de mouvement perpétuel. Il s'agirait aussi de croyances selon lesquelles, au-delà du seuil mystérieux des forêts, les chasseurs se métamorphosent en cerfs sous le signe du désir de liberté 9 . La montagne, la forêt et la source sont les symboles de cette liberté ${ }^{10}$. Selon une autre interprétation, la métamorphose est un voyage dans le mystère de l'autre monde ${ }^{11}$. Un catalogue de colinda mentionne deux causes possible : soit les fils du vieux chasseur ont bu dans l'empreinte du grand cerf, soit Dieu les a punis. Il est certain que nous assistons au fonctionnement d'un rituel de passage, prescrit pour des jeunes gens qui vont devenir adultes et se retirent dans la nature pour éviter les brutalités de leurs parents $^{12}$. La comparaison entre plusieurs variantes de la même colinda nous offre un ensemble d'explications rationnelles de la métamorphose. Dans la collection de Nicola, les causes sont multiples : malédiction des parents, punition infligée par le grand cerf ou par Dieu, parce que la chasse poussée a l'extrême est un attentat contre la nature. On assiste donc à une vision casuistique. Malgré cela, « le problème de la métamorphose reste ouvert ${ }^{13}$. » Quoi qu'il en soit, les deux colinda ne semblent offrir aucune motivation valable à cette idée singulière qui a permis à un auteur de conclure que les jeunes chasseurs, en étant changés en cerfs, se sont initiés à la vie monastique : qu'ils seraient devenu moines ${ }^{14}$.

En revanche, une autre interprétation peut être proposée, si l'on tient compte de la période de l'année au cours de laquelle les colinda étaient chantées. En l'occurence, au temps du solstice d'hiver : période dangereuse. Sol stare signifie que le sol s'arrête, et cela, plus grave encore : mundus patet ${ }^{15}$ - partout en Europe. Le monde des vivants est agressé par les morts qui reviennent. Pour les Fran- 
çais, la bûche qui se consume dans la cheminée était un acte de défense destiné à avertir les agresseurs. Pour les Roumains (ainsi que dans la vision de Bartók), la vieille mère a préparé la table avec du vin et du pain et elle attend le retour de ses fils à la lumière de la torche. Pour les Hongrois, on ne sait plus si l'on attend des anges ou des défunts ${ }^{16}$, ou alors les populations sondées restent-elles désespérément taciturnes face aux ethnographes. Parfois, tout de même, quelque initié veut bien nous renseigner. En 1965, dans le village de Taktaszáda, situé au nord de la Hongrie, un sacristain a révélé que le prêtre, à travers la serrure du tabernacle de l'autel, pouvait observer l'apparition de sorciers cornus ${ }^{17}$. Les revenants sont dangereux. Qui sont ces revenants? Les fils du vieux chasseur, dans la colinda de Bartók, ne sont-ils pas des revenants ? Une chose est sûre, ils ont disparus à jamais. La disparition est un évènement déplorable. « Dans les communautés primitives et sauvages, la séparation des parents et des fils est une tragédie personnelle, un accident sociologique »-écrit Malinowski ${ }^{18}$. La tragédie est aggravée dans le cas où les disparus sont restés adolescents, s'il n'ont pas vécu une vie conforme aux normes de la vie. Dans la culture roumaine, ils peuvent devenir des strigoi (une sorte de vampire). Les strigoi ne respectent pas le contrat passé entre les vivants et les morts dont a parlé Claude Lévi-Strauss ${ }^{19}$. Dès lors, les vivants doivent se défendre. Et, dans le cas des colinda qui nous intéressent, cet acte de défense est accompli par la métamorphose, ou, pour mieux dire, c'est la nature elle-même qui accomplit l'acte de défense à travers la métamorphose. La colinda est un mythe en action - écrit Ovidiu Bîrlea. " L'action ne se déroule pas dans le passé, elle est imminente, elle se déroule sous nos yeux à mesure que l'on chante la colinda, et cet acte peut être répété autant de fois que cela semble nécessaire. » Le temps du solstice d'hiver, en particulier, l'exige ${ }^{20}$. La performance de la colinda est un acte magique ${ }^{21}$, c'est-à-dire une croyance qui fait naître le miracle. En l'occurence, le vieux chasseur, malgré sa douleur, peut s'en retourner tranquillement chez lui, avec l'assurance que ses fils ne reviendront pas sous la forme de strigoi. Les angoisses ancestrales sont pacifiées, et cela de manière pacifique. Observons au passage le contraste avec un mythe bororo dans lequel un fils changé en cerf, au contraire, tue son père ${ }^{22}$.

Être un cerf dans la mythologie peut signifier beaucoup de choses. Bartók s'est amusé à transcrire des colinda, mais il ne s'est jamais vraiment aventuré sur le terrain mythologique. Il semble avoir étudié avec attention un livre sur la colinda roumaine dans lequel se trouve un avertissement pour les mythomanes : « Le problème du culte du cerf est très complexe, et plus l'exégète s'efforce de lui trouver une solution, plus il s'abîme dans un terrain marécageux où le cerf lui-même disparaît et nous à sa suite ${ }^{23} »-c^{\prime}$ 'est dans le marais de l'histoire qu'est né le discours savant sur la Cantata profana.

Ce discours, au demeurant, a atteint son apogée avec l'analyse de Károly Kerényi, en 1949. Kerényi était un savant réputé spécialisé dans la mythologie antique. 
Sa correspondance avec Thomas Mann a été plusieurs fois rééditée. Après la guerre, il fut interdit de retour par les autorités communistes (il résidait en Suisse). En ce qui concerne la Cantata profana, il s'est gardé de spéculer à outrance au sujet de la métamorphose. Mais il savait très bien que dans le monde antique romain, le cerf était le symbole de l'esclave fugitif. Pour lui, la Cantata était donc le chant de l'exode, un acte de protestation contre la civilisation criminelle ${ }^{24}$. L'article dans lequel Kerényi proposait cette interprétation ne fut publié que partiellement en Hongrie et seulement dans les années 1960. Ironie de l'histoire : une seule et même personne, György Alexics, communiste et mathématicien de renom, d'une part, fut à l'origine de l'interdiction de territoire opposée à Kerényi en 1949 et, d'autre part, vers la fin des années 1930, accorda une aide substantielle à Bartók pour la transcription des chants populaires roumains. Alexics ne l'a pas nié : le penseur humaniste qu'était Kerényi aurait pu être un adversaire gênant. Il était, en revanche, plus commode de se vanter d'avoir eu l'amitié de Bartók en oubliant un moment inconfortable : car le même Alexics avait aussi participé à la Commune de Budapest, en 1919, avant de se réfugier en Roumanie où il avait accusé Bartók de falsifier le folklore roumain en exagérant le rôle des influences hongroises ${ }^{25}$. Non moins paradoxal est le cas de György Lukács, le plus important théoricien marxiste du $\mathrm{XX}^{\mathrm{e}}$ siècle. Lukács a attaqué Kerényi en l'accusant de favoriser une atmosphère de mythes rétrogrades. Dans le même temps, il a réfuté la critique d'Adorno reprochant à Bartók son idéalisation de la nature et son folklorisme irrationnel. À toute fin utile, n'oublions pas qu'il y a eu plusieurs Lukács. L'un, le jeune, était parmi les créateurs de l'atmosphère d'où est sortie la Cantata. L'autre, le vieux Lukács, plus ou moins désabusé, se retrouvait peut-être encore dans la Cantata, mais d'une manière ambiguë.

Le caractère taciturne de Bartók est bien connu. Dans ses écrits, il s'est toujours exprimé de manière laconique. Le hinterland verbal de son œuvre se retrouve dans les conversations infinies de ses contemporains, jeunes gens qui formaient le Cercle de dimanche, dont l'animateur était précisément Lukács. Une douzaine de philosophes et d'écrivains se rassemblaient dans le deux pièces de Balázs Béla, tous en quête des certitudes métaphysiques et avec le dessein de créer une communauté socio-spirituelle. Ces réunions au caractère quasi-religieux se tenaient sous le signe de la sincérité - selon le souvenir d'Anna Lesznai. Bartók n'y participa que quelques fois, sans dire un mot. Avec son sourire souple, il exprimait à la fois sa sympathie et sa méfiance à l'égard des divagations métaphysiques et gnostiques dont Lukács était le maître incontesté, jusqu'à l'exaspération finale, quand, vers la fin de sa vie, ce dernier a fini par confesser : "Ich bin eine gescheiterte Existenz ». Le grand problème qui tourmentait Lukács était celui de la dissonance entre l'âme et la forme, c'est-à-dire celui de l'aliénation. Il en a trouvé l'antidote (l'union de l'âme et de la forme) dans la révolution communiste, entendu que le Parti incarne l'âme tandis que la classe ouvrière représente la forme. Lors de 
la Commune de Budapest, en 1919, Lukács, devenu Commissaire du peuple à la culture, a offert à Bartók une place à la Direction pour la musique. La Commune offrait l'espoir de sortir le pays du chaos général dans lequel il se trouvait et, plus spécifiquement, de créer des institutions efficaces pour l'enseignement musical. On est en droit de se poser la question suivante : dans quelle mesure Bartók a-t-il été tenté par la gnose?

Dans les écrits de Bartók, le mot de gnose n'est jamais mentionné. Or l'intrigue de son opéra, le Château de Barbe-bleu, a bel et bien pour fondement un mythe gnostique. La lumière entre dans les ténèbres et se consume - c'est ainsi que Béla Balázs a résumé son propre livret ${ }^{26}$. Au demeurant, l'œuvre précisément démasque le mensonge gnostique : sans l'intermédiaire social, la confrontation entre l'individu et la nature mène à la tragédie ${ }^{27}$. Pour les gnostiques, la nature est mauvaise. D'après un Lukács ou un Cioran, la rédemption a nécessairement pour corollaire une modification de la nature. D'après Bartók, au contraire, c'est dans la nature, telle quelle, que se trouve la rédemption. Du reste, les analyses savantes tendent à exagérer sa religion de la nature, quasiment panthéiste, et la spirale verbale atteint son point culminant dans le dicton Credo quia absurdum. Mais ce dernier est-il pertinent si on le rapporte à la Cantata profana et à la vision de monde de son auteur?

Bartók s'est peu livré au sujet de ce qu'il croyait. Ses lectures, ou mieux : ses annotations, nous offrent quand même une sorte de tableau d'ensemble. Lire, pour lui, était un dialogue. Et ses dialogues sont le témoignage d'une évolution qui a conduit jusqu'à la Cantata profana. Nietzsche, Rousseau et Ady présidaient à ces dialogues. Nietzsche a catalysé la démolition des valeurs traditionnelles. Il a été à l'origine d'une crise profonde. Bartók faisait la cour à une jeune violoniste en lui récitant des passages de la sagesse de Zarathustra ; de cette manière, l'insuccès était garanti. De fait, le jeune compositeur fut tenté par l'idée du suicide. Il est arrivé à la limite du néant, selon les mots de son ami Zoltán Kodály. Puis Bartók a mis un terme à la lecture de Nietzsche. Ses dernières notes soulignent le passage suivant : «Tu dois être ce que tu $\mathrm{es}^{28}$. » La dialogue avec Rousseau nous présente ce qu'était alors Bartók : désaccord sur le terrain musical, accord dans le culte de la nature : « Jamais la nature ne nous trompe; c'est toujours nous qui nous trompons. » Bartók a marqué cette maxime avec un signe d'exclamation dans la marge ${ }^{29}$. Il a signalé aussi l'éloge de l'homme naturel : « Le bonheur de l'homme naturel est aussi simple que sa vie ; il consiste à ne pas souffrir : la santé, la liberté, le nécessaire le constituent ${ }^{30}$. » La notation suivante nous conduit au noyau de la Cantata: «c'est dans le cœur de l'homme qu'est la vie du spectacle de la nature ; pour le voir, il faut le sentir ${ }^{31}$. »

D'après Bartók, résident d'une grande ville tourmenté par des obsessions suicidaires, la poésie d'Endre Ady a été une planche de salut. Elle lui démontrait que l'on pouvait être à la fois moderne et classique. Les volumes d'Ady incitaient 
à créer une mythologie nouvelle, en l'attente d'une révolution démocratique. Bartók s'est en quelque sorte identifié au poète Ady, qui « était tout et le contraire de tout »-comme l'a remarqué Lukács ${ }^{32}$. Tout était donc possible, comme dans le monde des contes.

Le conte, d'ailleurs, était une préoccupation permanente au sein du Cercle du dimanche. Plus tard, Béla Balázs a écrit des contes appréciés par Thomas Mann. Les contes d'Anna Lesznai ont été, quant à eux, récemment réédités. D'après Lukács, qui était à l'origine de cette obsession et en a fait la théorie, ce n'est pas l'invention qui constitue la nouveauté du conte, mais la manière particulière de redécouvrir l'unité cosmique primordiale. Un conte, on le contemple, mais on vit aussi en lui, en dépit de son caractère féerique. Car il lance un appel en provenance du monde de l'au-delà : il exprime des réalités spirituelles non-euclidiennes et en cela identiques à celles de l'homme moderne. Le mythe offre le spectacle des racines inconnues de nos réalités, le conte est une allégorie dont le sens est perdu à jamais, toutefois son caractère infini permet-il d'attirer l'attention sur le caractère lui aussi infini de l'âme ${ }^{33}$. C'est le résumé d'une poésie conceptuelle, non sans aspirations politiques.

Lukács, arrivé au pouvoir en 1919, a lancé un concours de conte moral. On peut considérer la Cantata profana comme une hypostase nouvelle de ce culte du conte par une jeunesse tumultueuse. En 1930, Bartók réactivait en quelque sorte ces anciennes préoccupations. En réalité, il envisageait un triptyque dont la Cantata aurait été un élément. Dans ce triptyque, trois peuples, en l'occurence hongrois, roumain et slovaque devaient rivaliser pour la primauté. Et le jugement de Dieu, créateur de toute choses, aurait été rendu de la manière suivante : «Celui qui a la création la plus belle aura la primauté34 ${ }^{34}$ »L'histoire réelle a pris de relais de cette histoire imaginaire. Et la Cantata nous est restée comme la contribution personnelle de Bartók à cette histoire réelle.

Quelle contribution? Quelle sorte de message ? La Cantata suggère-t-elle de croire en l'absurde ? Au contraire, le dicton déjà cité dit autre chose : ce n'est pas en l'absurde que je crois, au contraire, le monde est absurde, si je ne crois ce que je dois croire ${ }^{35}$. Il est à la mode de mettre en parallèle Jésus et les fils changés en cerfs. On peut invoquer l'Évangile selon Matthieu, et naturellement les versets marqués par Bartók : « Mon Père, s'il est possible, que cette coupe s'éloigne de moi ! » (26:39) Et aussi : « Je vous le dis, je ne boirai plus désormais de ce fruit de la vigne, jusqu'au jour où j'en boirai du nouveau avec vous dans le royaume de mon Père. » (26:29) ${ }^{36}$ L'allusion musicales à la Passion selon Saint Matthieu de Bach pourraient symboliser la transfiguration ou la transsubstantiation des fils et leur liberté ultime ${ }^{37}$. La parallèle entre la métamorphose des fils et la résurrection de Jésus nous renvoie vers un monde sans péché ${ }^{38}$. Ces spéculations sont bien sûr à mettre au conditionnel, mais, dans une extase artistique, tout est possible. Au demeurant, Bartók a vu en Jésus un moralisateur, le créateur d'une 
des « plus grandes œuvres existantes et dont les leçons peuvent, à plus d'un titre, nous épater ${ }^{39}$. » Par exemple, dans sa Bible en espagnol, Bartók a posé un signe d'interrogation en marge des versets suivants : « Ne croyez pas que je sois venu apporter la paix sur la terre ; je suis venu apporter, non la paix, mais le glaive. Je suis venu mettre la division entre le fils et son père, entre la fille et sa mère, entre la belle-fille et sa belle-mère. » (Matthieu 10:34-35) Ce signe d'interrogation invalide la thèse de Lukács, pour qui la Cantata serait la rupture totale avec le monde des pères, avec le monde bourgeois. L'exode des fils est une tragédie, y compris pour eux-mêmes. Une Imitatio Christi à proprement parler est invalide parce que les fils changés en cerfs ont menacé leur père. Et, ne l'oublions pas : Bartók a aussi lu l'Ancien Testament. Dans sa Bible en roumain, il a noté dans la marge, en hongrois, la traduction des mots inconnus, là où le Livre de Josué décrit l'occupation de la terre promise ${ }^{40}$. La forêt des fils n'est-elle pas une autre terre promise ? Nous savons que Béla Bartók a changé de confession. Il a quitté le catholicisme et s'est converti à l'unitarisme, dont la fondateur, Ferenc Dávid, a milité en son temps pour la fondation d'une nouvelle Jérusalem, pays de Dieu. Bartók, avec une amertume pragmatique, a constaté combien Dieu s'était retiré du monde. Le caractère infini de l'univers lui a procuré le delirium tremendi de sentiments religieux plus ou moins panthéistes, tels qu'ils s'expriment dans la Cantata profana. Et rien ne caractérise mieux cette œuvre qu'un verset d'Endre Ady, souligné par Bartók lui-même, et qui donne à la poésie son autodéfinition :

« Malédiction, psaume, lamentation et chant. »

\section{Notes}

1 La base de cet article est mon livre „Tiszta forrás” felé... Közelitések Bartók Béla és a Cantata profana világához [Vers la "source pure"... Réflexions sur le monde de Béla Bartók et des Cantata Profana], Budapest, Gondolat, 2011

2 Tallián Tibor, Cantata profana - az átmenet mítosza [Canta profana - le mythe de la transition], Budapest, Magvetö, 1983, p. 212

3 Bartók Péter, Apám [Mon père], Budapest, Editio Musica, 2004, p. 239

4 Ioan R. Nicola, „Colinda vînătorilor metamorfozaţi în cerbi” [La colinda des chasseurs transformés en cerfs], Lucrări de muzicologie, vol. IV, Cluj-Napoca, 1968, pp. 59-86

5 Babits Mihály, Elöszó. in Új Anthológia. Fiatal költők 100 legszebb verse, Nyugat, Bp, 1932, p. 12

6 Bartók Béla, Összegyüjtött írásai [Oeuvre réunies], Szerk. Szőllősy András, vol. I, Budapest, Zenemúkiadó, 1966, p. 600

7 Bartók Béla, Válogatott írásai [Oeuvres choisies], Szerk. Szőllősy András, Budapest, Müvelt nép, 1956, p. 213

8 Bartók Béla levelei [Lettres de Béla Bartók], Demény János (dir), Budapest, f.e., 1976, p. 398

9 Mihai Pop, Pavel Ruxăndoiu, Folclor literar românesc [Le folklore littéraire roumain], București, Ed. Didactică și Pedagogică, 1976, pp. 170-171 
10 Pavel Ruxăndoiu, Folclorul literar în contextul culturii populare româneşti [Le folklore littéraire dans le contexte de la culture populaire roumaine], București, Ed. Grai și suflet, Col. „Cumințenia pămîntului”, 2001, p. 260

11 Vasile V. Filip, Universul colindei româneşti [L'univers des colinda (chants populaires) roumains], București, Ed. Saeculum, 1999, p. 156. Rezumă articolul său din 1979

12 Monica Brătulescu, Colinda românească [les colinda roumaines], București, Minerva, 1981, pp. $50-55,62,216$

13 Nicola, op.cit., p. 76

14 Sabina Ispas (red.), Colindatul tradiţional românesc. Sens şi simbol [Les colinda traditionnelles roumaines. Sens et symbole], Bucureşti, Saeculum vizual, 2007, pp. 267-268

15 E. Schneeweiß, Serbokroatische Volkskunde [Le folklore serbo-croate], Berlin, De Gruyter, 1961, p. 124

16 Dömötör Tekla, A magyar nép hiedelemvilága [le monde des croyances populaires hongroises], Budapest, Corvina, 1981, p. 219

17 Szabó Lajos, Taktaszádai mondák [la légende de Taktaszád], Budapest, Akadémiai Kiadó, 1975, p. 174.

18 Bronisław Malinowski, Sex and Repression in Savage Society, London/New York, Routledge, [1927], 2001, p. 182

19 Claude Lévi-Strauss, Tristes tropiques, Paris, Plon, 1961, p. 242

20 Ovidiu Bîrlea, Folclorul românesc [Le folklore roumain], vol. I, Bucureşti, Minerva, 1981, pp. 326-327

21 Petru Caraman, Colindatul la români, slavi şi la alte popoare. Studiu de folclor comparat [Chants roumains, slaves et d'autres peuples. étude de folklore comparé], Bucureşti, Minerva, 1982

22 Claude Lévi-Strauss, Le cru et le cuit, Paris, Plon, 1964, p. 148

23 Schmidt Tibolt, A hazai oláhság kolinda-költészete [Le chant et la poésie chez les Roumains de Hongrie], Budapest, Fritz, 1913, p. 27

24 Karl Kerényi, Wege und Weggenossen [Routes et compagnons de route], vol. 2, München,Langer, 1988, pp. 364-370

25 G. G. Alexici, « Elemente române în musica populară maghiară » [Élément roumain dans la musique populaire hongroise], în Grai şi suflet, nr. 1, 1927, pp. 47-81

26 Lukács György levelezése (1902-1917) [Correspondance de...], Budapest, Magvető, 1981, p. 638

27 Ujfalussy József, Bartók Béla, Budapest, Gondolat, 1976, p. 148

28 Denijs Dille, Béla Bartók. Regard sur le passé, Namur, Presses Universitaires de Namur, 1990, p. 98

29 Rousseau, Jean-Jacques, Émile ou de l'Éducation, vol. II, Paris, Librairie de la Bibliothèque Nationale, 1903, p. 130. BA=Bartók Archívum

30 Ibid., vol. I, p. 81

31 Ibid.

32 Lukács György, «Ady Endre minden és mindennek az ellenkezője » [Endre Ady, adversaire de tout et de tous], in Lukács György, Ady Endréröl, Budapest, Magvető, 1977, p. 14

33 Id., Ifjúkori müvek, Budapest, Magvető, 1977, pp. 710-724; Lukács György, Balázs Béla és akiknek nem kell, [Béla Balázs et ceux qui ne veulent pas de lui], Gyoma, Kner, 1918, pp. 103-121

34 Bartók breviárium, Újfalussy József, Lampert Vera (dir), Budapest, Zenemükiadó, 1980, pp. 345-347

35 Henri H. Stahl, Eseuri critice despre cultura populară românească [Essais critiques sur la culture populaire roumaine], Bucureşti, Minerva, 1983, p. 188 
36 Tallián Tibor, «...múljék el e pohár tőlem...» [Éloignez de moi cette coupe], Magyar zene, nr. 2, 1999, pp. 153-159

37 Benjamin Suchoff, Béla Bartók. Life and Work, London, Scarecrow Press, 2001, p. 113

38 Elliot Antokoletz, « Modal Transformation and Musical Symbolism in Bartók's Cantata profa$n a »$, Bartók Perspectives. Elliot Antoloketz, Victoria Fischer, Benjamin Suchoff (dir), Oxford, Oxford University Press, 2000, p. 75

39 Bartók Béla levelei [Correspondance de...], Szerk. Demény János, Budapest, f.e, 1976, p. 125

40 Sânta Scriptură a Vechiului şi Noului Testament [Les Saintes Écritudes de l'Ancien et du Nouveau Testament], Tipărit cu spesele Societăţii Biblice Britanice şi Străine, Bucuresci, Strada Salcâmilor 2, 1909, pp. 2-4. BA 